\title{
Publisher's Note: Crucial role of interlayer distance for antiferromagnet-induced perpendicular magnetic anisotropy [Phys. Rev. B 92, 214435 (2015)]
}

\author{
Bo-Yao Wang, Po-Han Lin, Ming-Shian Tsai, Chun-Wei Shih, Meng-Ju Lee, Chun-Wei Huang, Nae-Yeou Jih, \\ Pei-Yu Cheng, and Der-Hsin Wei \\ (Received 25 May 2016; published 6 June 2016)
}

DOI: 10.1103/PhysRevB.93.219901

This paper was published online on 28 December 2015 with an error in Fig. 3. Figure 3 has been replaced as of 25 May 2016. The figure is incorrect in the printed version of the journal; therefore for the benefit of the print readership, the figure and its caption are replicated below.
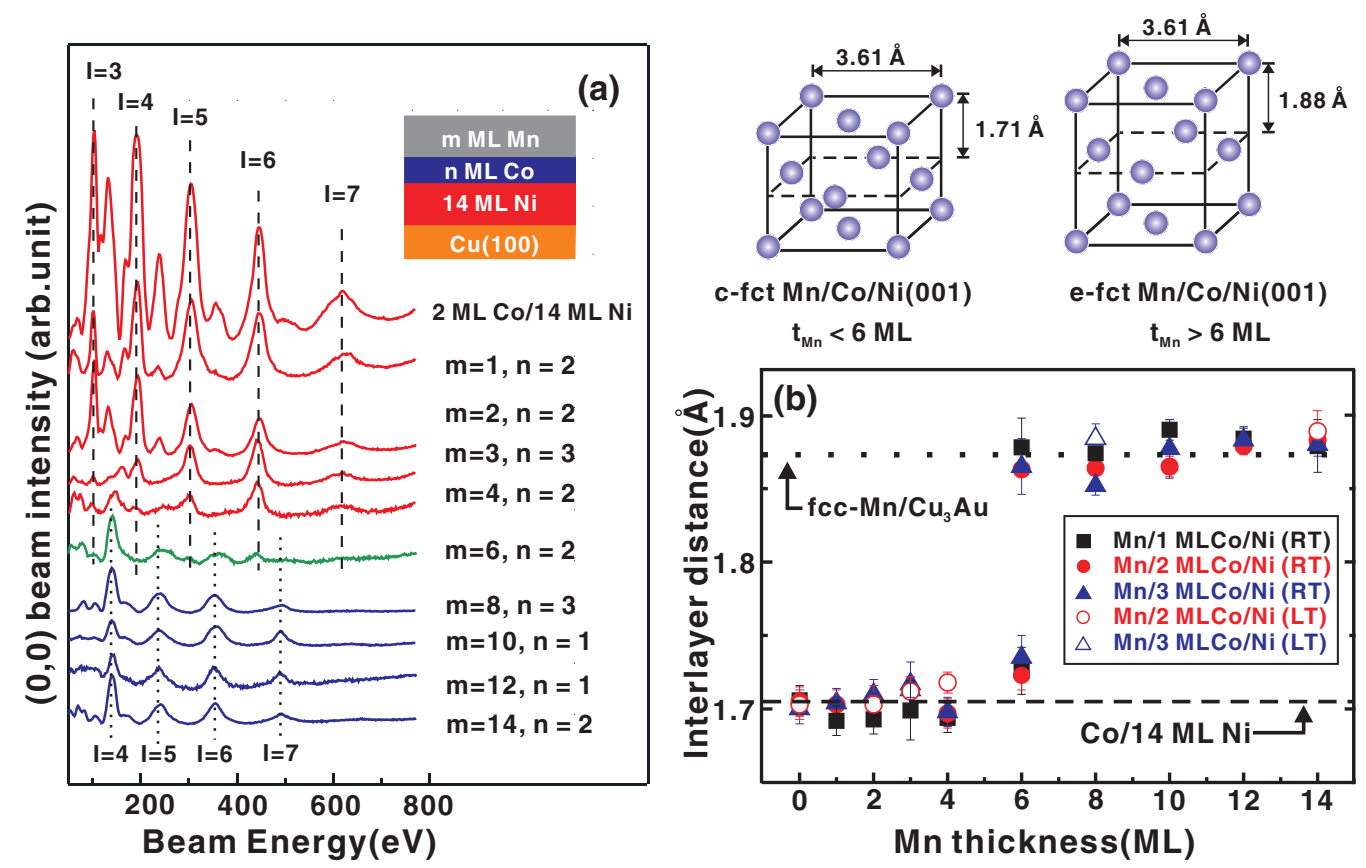

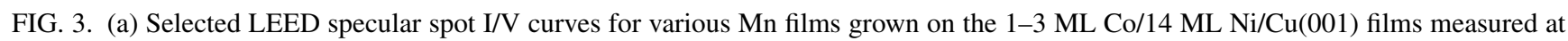
$300 \mathrm{~K}(\mathrm{RT})$. (b) Average interlayer distance $\left(d_{\perp}\right)$ of various films calculated according to the energy peaks (I) in the I/V curves at RT or $150 \mathrm{~K}$ (LT). The $d_{\perp}$ of the Mn films changed from approximately 1.71 to $1.88 \AA$ when $t_{\mathrm{Mn}}>6 \mathrm{ML}$. In (b), the dashed line at the bottom represents $d_{\perp}$ of the 1-3 ML Co films grown on $\mathrm{Cu}(001)$; the dotted line at the top shows the $d_{\perp}$ of the fcc-Mn films grown on $\mathrm{Cu} 3 \mathrm{Au}(001)$ [11]. The crystalline structures of the c-fct Mn and e-fct Mn films, determined from the LEED and LEED I/V analysis, are shown at the top. 